\title{
Turbulent optimization of toroidal configurations
}

H. Mynick ${ }^{1}$, P. Xanthopoulos ${ }^{2}$, B. Faber ${ }^{1}$, M. Lucia ${ }^{1}$, M. Rorvig ${ }^{3}$, and J.N. Talmadge ${ }^{3}$

${ }^{1}$ Plasma Physics Laboratory, Princeton University, Princeton, NJ

${ }^{2}$ Max-Planck-Institut für Plasmaphysik, EURATOM Association, Greifswald, Germany

${ }^{3}$ Depts.of Physics and Electrical Engineering, University of Wisconsin, Madison, WI

Recent progress in "turbulent optimization" of toroidal configurations is described, using a method recently developed for evolving such configurations to ones having reduced turbulent transport. The method uses the GENE gyrokinetic code to compute the radial heat flux $Q_{g k}$, and the STELLOPT optimization code with a theory-based "proxy" figure of merit $Q_{p r}$ to stand in for $Q_{g k}$ for computational speed. Improved expressions for $Q_{p r}$ have been developed, involving further geometric quantities beyond those in the original proxy, which can also be used as "control knobs" to reduce $Q_{g k}$. Use of a global search algorithm has led to the discovery of turbulent-optimized configurations not found by the standard, local algorithm usually employed, as has use of a mapping capability which STELLOPT has been extended to provide, of figures of merit over the search space.

\section{INTRODUCTION}

Since the inception of the fusion program, transport due to plasma turbulence has been a major challenge. For the past 3 decades, refined shaping of stellarators has been employed to develop "transport-optimized" designs which greatly reduce the expected neoclassical (NC) transport, to levels typically subdominant to the expected turbulent levels, making reducing turbulent transport in both stellarators and tokamaks the prime target for further confinement enhancement. Until the past few years, however, while there has been much study of turbulent transport mechanisms in toroidal devices, the notorious complexity of plasma turbulence has prevented also using shaping as a means of designing toroidal systems for reduced turbulent transport. However, the recent advent of two powerful numerical tools has made this goal feasible, namely, configuration optimization codes such as STELLOPT[1], and nonlinear gyrokinetic (GK) codes valid for 3D configuations, such as GENE[2, 3]. Using 
these, we have developed a method $[4,5]$ which for the first time permits designing configurations for reduced turbulent transport. Such a capability has the potential to considerably improve the attractiveness of fusion via magnetic confinement.

STELlopt seeks to minimize a cost function $C^{2}(\mathbf{Z})$ in the search space $\mathbf{Z} \equiv\left\{Z_{j}\right\}$ specifying the shape of the configuration (e.g., the $Z_{j}$ may be the Fourier amplitudes specifying the shape of the plasma boundary). To evolve configurations having reduced turbulent transport, one needs a turbulent contribution $C_{t}^{2}$ to $C^{2}$, a figure of merit (FOM) which is larger the larger the level of turbulent transport. As discussed[4], ideally for $C_{t}$ one could use the radial heat flux $Q_{g k}$ from nonlinear GK runs, but this is far too computationally expensive. To overcome this, the method instead uses a "proxy" function $Q_{p r}(\mathbf{Z})$ to stand in for $Q_{g k}(\mathbf{Z})$, which uses the same geometric input information as the GK run would, but is far faster to compute. After STELLOPT has evolved such a configuration in Z-space using $Q_{p r}$, the method's second step confirms that the resultant configuration in fact has reduced $Q_{g k}$ by doing a nonlinear GENE run on it. Of course, this procedure works better the more closely the topography in $\mathbf{Z}$-space of $Q_{p r}$ and $Q_{g k}$ resemble each other, so that the trajectory toward lower $Q_{p r}$ which Stellopt follows also tends to lower $Q_{g k}$. Thus, differing forms for $Q_{p r}$ will guide STELlOPT toward differing evolved configurations. To obtain a $Q_{p r}$ resembling $Q_{g k}$, the proxies we have used are theory-based expressions, either for the actual heat flux $Q$, or for some quantity expected to scale with $Q$, such as the growth rates $\gamma_{\mathbf{k}}$ of the modes comprising the turbulence. A perfect match between $Q_{p r}$ and $Q_{g k}$ is more than one can expect, tantamount to having a complete and correct theory of turbulent transport. However, the fairly simple expressions used thus far (see Sec. II) have enjoyed substantial success, and as discussed here, improved forms are being found.

We note that the optimizations done here are only on the shape of the device, i.e., only in Z-space, akin to what has been done in developing NC transport-optimized stellator designs, and not on plasma profile quantities such as gradients in temperature or density. Given a device with a specified shape, the further issues of obtaining self-consistent profiles, and what profiles would optimize device performance are important, but outside the scope of this work.

The initial success of the method has led to an exploration of its possibilities. Starting with the NCSX (National Compact Stellarator Experiment)[6] quasi-axisymmetric (QA) stellarator design, [4] followed by a closely related D-shaped tokamak, [5] STELLOPT with the 
original $Q_{p r}$ (designated "proxy-1" or $Q_{p r 1}$ ) produced configurations reducing the level of ion temperature gradient (ITG) turbulent transport by factors of $2-3$. The method was less successful with other confinement classes, however.[7] From the HSX (Helically Symmetric Experiment)[8] quasi-helical (QH) design, a significant but less dramatic reduction in $Q_{g k}$ of about $23 \%$ was obtained, while for the W7X (Wendelstein 7-X) [9] quasi-omnigenous/quasiisodynamic (QO/QI) design, little improvement was achieved. Concomitantly, the agreement of the prediction $Q_{p r}(z)$ along a field line (parametrized by parallel coordinate $z$ ) with $Q_{g k}(z)$ for these 4 device classes was found to be least satisfactory for the QO/QI class. As discussed here, subsequent experimentation with $Q_{p r}$ has led to a small set of improved, more first-principles proxy functions. The most effective of these to date ("proxy-1d", or $\left.Q_{p r 1 d}\right)$ is also the variant which manifests the best agreement between $Q_{p r}(z)$ and $Q_{g k}(z)$. Proxy-1 succeeded principally by boosting $\kappa_{1} \equiv \mathbf{e}_{r} \cdot \boldsymbol{\kappa}$, the radial component of the vector curvature $\boldsymbol{\kappa}$, with $\mathbf{e}_{r}$ the covariant basis vector for minor radial coordinate $r \equiv\left(2 \psi / B_{a}\right)^{1 / 2}$, $2 \pi \psi$ the toroidal flux, and $B_{a}$ the average magnetic field strength at the plasma edge, where $r=a$. The new proxies retain this, but involve additional geometric quantities which can be modified via shaping to substantially reduce $Q_{g k}$. Thus, this paper extends not only the range of toroidal configurations which have been explored, but also the number of geometric "control knobs" at one's disposal to modify the turbulent transport.

The method has been enhanced by the use of a global, "differential evolution" (DE) search algorithm [alternate to STELLOPT's standard, local Levenberg-Marquardt (LM) algorithm], originally implemented in STELLOPT to facilitate finding QAs with reduced NC transport[10], but only recently applied to the turbulent-optimization challenge.[11] In conjunction with an improved proxy close to $Q_{p r 1 d}$, this algorithm enabled the discovery of the first W7X-derived configuration having appreciably reduced $Q_{g k}$.

A further extension of the method has been achieved by using a mapping capability implemented in Stellopt. [7] Here we use this capability to not only visualize the variation of FOMs over the search space $\mathbf{Z}$, but using this, to find configurations with lower values of the cost function $C^{2}(\mathbf{Z})$ than found previously with either search algorithm.

The remainder of this paper is organized as follows. In Sec. II we introduce some useful notation and coordinate systems, and discuss the turbulent proxy functions we have employed. In Sec. III we describe configurations obtained using these, using the 2 search algorithms and the mapping capability. We also describe our efforts thus far to obtain con- 
figurations satisfying not only the objective of improved turbulent transport, but also other constraints needed for a practical device one might consider building. Finally, we provide a summarizing overview in Sec. IV.

\section{PROXY FUNCTIONS}

It is convenient to parametrize a torus with straight field-line (flux) coordinates $(\psi, \theta, \zeta)$, with poloidal and toroidal azimuths $\theta$ and $\zeta$ satisfying $\mathbf{B}=\nabla \psi \times \nabla \theta+\nabla \zeta \times \nabla \psi_{p}=\nabla \psi \times \nabla \alpha$, with magnetic field $\mathbf{B}$, toroidal flux $2 \pi \psi$, poloidal flux $2 \pi \psi_{p}$, binormal azimuth $\alpha \equiv \theta-\zeta \iota$, and rotational transform $\iota(\psi) \equiv d \psi_{p} / d \psi$. Defining wavevector $\mathbf{k} \equiv k_{\alpha} \nabla \alpha$ makes $\mathbf{k}$ normal to $\mathbf{B}$, i.e., $\mathbf{k} \cdot \mathbf{B} \equiv k_{\alpha} \nabla \alpha \cdot \mathbf{B}=0$. To parametrize the flux-tube domain of its simulations, GENE uses the closely related local coordinate system[3] $\left(x \equiv r-r_{0}, y, z\right)$, with units of length in the radial, binormal, and parallel directions, respectively, with corresponding unit vectors $(\hat{x} \equiv \hat{r} \equiv \nabla \psi /|\nabla \psi|, \hat{y} \equiv \hat{b} \times \hat{x}, \hat{z} \equiv \hat{b} \equiv \mathbf{B} / B)$, and $r_{0}$ the $r$-value on which the tube is centered.

As in Ref. 4, the radial heat flux $Q_{s}$ for species $s$ may be written

$$
Q_{s}=-\chi_{s} g^{r r} n_{0} d T_{s} / d r
$$

with $\chi_{s}$ and $T_{s}$ the thermal conductivity and temperature for species $s$. Here, $g^{r r} \equiv g^{x x}$ is the $\operatorname{rr}$ component of metric tensor $g^{i j} \equiv \nabla q^{i} \cdot \nabla q^{j}$, for any 2 coordinates $q^{i}, q^{j}$. Using a quasilinear model for $\chi_{s}$ plus a simple mixing-length assumption for the saturation level of the turbulent amplitudes $\phi_{k}$, one finds $\chi_{i}=\sum_{\mathbf{k}} D_{\mathbf{k}}$, with $D_{\mathbf{k}} \simeq c_{D} \gamma_{\mathbf{k}} / k_{\perp}^{2}$. Using a simplified dispersion equation for ITG modes with adiabatic electrons (ITG-ae), in Ref. 4 we found growth rate $\gamma_{\mathbf{k}} \simeq\left(\omega_{* i} / \kappa_{n}\right)\left|\tau \kappa_{1}\left(\kappa_{p}-\kappa_{c r}\right)\right|^{1 / 2} H\left(\kappa_{p}-\kappa_{c r}\right) H\left(-\kappa_{1}\right)$. Here one sees the appearance of the radial curvature $\kappa_{1}$ noted in Sec. I, negative for "bad" curvature, and positive for "good" curvature, $H(\kappa)$ is the Heavyside function, $\omega_{* i}$ is the ion diamagnetic frequency, $\kappa_{n} \equiv L_{n}^{-1} \equiv-\partial_{r} \ln n_{0}$, and $\kappa_{p} \equiv \kappa_{n}+\kappa_{T}$, where $\kappa_{T} \equiv-\partial_{r} \ln T_{i}$. In Ref. 4 the critical pressure gradient $\kappa_{c r}$ and multiplicative constant $c_{D}$ were determined by a best fit of $Q_{p r}$ to the GK results for a set of flux tubes on a family of toroidal configurations studied earlier[12]. Finally, the factor $g^{r r}$ appearing in (1) above was dropped in the original $Q_{p r 1}$ used in Stellopt from the expectation that it would have little effect on the outcome. However, reducing $g^{r r}$ corresponds geometrically to increasing the distance between flux 
surfaces, which one might expect to weaken the instability, and experimentation with $Q_{p r}$ has shown that including this factor appreciably improves the agreement between $Q_{p r}$ and $Q_{g k}$, as well as its effectiveness in finding good configurations.

Not included in Eq.(1) are additional terms accounting for, e.g., gradients in density or rotational flow. Since as noted in Sec. I the optimizations done here are only on the shape of the device, these profile quantities are taken as fixed. Since we are here focussed on ITG turbulence, as in Refs. 4, 5 and 12, we take values of these profile quantities likely to drive ITG modes, with numerical parameters $a \kappa_{n}=0, a \kappa_{T}=3$, and $\tau \equiv T_{e} / T_{i}=1$ at the surface $r_{0} / a \simeq 0.7$, on which the optimization is chosen to center. For ITG-ae turbulence and for most stellarators, these gradient values are far above the critical gradients. (For $a \kappa_{n}=0$ the critical $a \kappa_{T}$ is typically around 1-1.3.)

In addition to the key geometric parameter $\kappa_{1}, Q_{p r 1}$ used in Ref. 4 had a dependence on the local shear $s_{l} \equiv \partial_{\theta}\left(g^{r y} / g^{r r}\right)$, entering via the factor $k_{\perp}^{2}$ in $Q_{p r}$, as $k_{\perp}^{-2}\left(s_{l}\right) \simeq \rho_{i}^{2}+\rho_{i} L_{p} /[1+$ $\left.\left\langle\left(\tau_{s} s_{l}\right)^{2}\right\rangle_{\Delta \theta}\right]$. This form arose from the intuition that $s_{l}$ plays a role similar to that played by flow shear, having a suppressive effect on the turbulence. While some GENE simulations have borne this intuition out, the particular dependence taken does not hold in general, tending to mislead STELLOPT toward configurations with large $s_{l}$, but for which the $Q_{g k}$ was not reduced. Removing this factor gives a variant "proxy-1b" of proxy-1, $Q_{p r 1 b}=\left\langle\gamma_{\mathbf{k}}\right\rangle$, which proved comparably effective to $Q_{p r 1}$ in generating configurations with reduced ITG transport. (Here, the angular brackets denote an average over a flux surface and sum over k.)

Instead of the $s_{l}$-dependent form for $k_{\perp}^{2}$ used in $Q_{p r 1}$, a more rigorous expression is the eikonal form from ballooning theory $k_{\perp}^{2}=k_{y}^{2} g^{y y}$, from the expression for $\mathbf{k}=\mathbf{k}_{\perp}$ given above. Using this and including the $g^{r r}$ factor already noted yields $Q_{p r 1 c} \equiv\left\langle\gamma_{\mathbf{k}} g^{r r} / g^{y y}\right\rangle$. While theory-based, this and the previous forms for $Q_{p r}$ are all somewhat heuristic, because the simple mixing-length assumption used in obtaining them is only a rough means of approximating the complicated nonlinear physics determining the saturation amplitudes. Thus, these and similar variants we have tested, such as $Q_{p r 1 d} \equiv\left\langle\gamma_{\mathbf{k}} g^{r r}\right\rangle, Q_{p r 1 e} \equiv\left\langle\gamma_{\mathbf{k}} / g^{y y}\right\rangle$, or $Q_{p r 1 f} \equiv\left\langle\gamma_{\mathbf{k}}\right\rangle /\left\langle g^{y y}\right\rangle$, are all comparably justified from an analytic standpoint, and which proxy to use derives from which produces results best agreeing with $Q_{g k}$ from simulations. In Fig. 1 is shown the comparison for $Q_{p r 1 d}(z)$ with $Q_{g k}(z)$. $Q_{p r 1 d}$ largely removes the disparity in amplitude for tubes- 1 and 2 for W7X for $Q_{p r 1}$ (see Ref. 4, Fig.1), without degrading the 
agreement of $Q_{p r}$ for the other tubes. The agreement for the surface-averaged values of $Q_{g k}$ and $Q_{p r}$ used by STELLOPT are somewhat better than the pointwise comparison shown here, because the regions of positive and negative difference tend to cancel. The full set of proxies discussed in this work are $Q_{p r 1}$, its variants $Q_{p r\{1 b-1 f\}}$ just introduced, $Q_{p r 1 d}$ 's close cousin $Q_{p r 1 x}$ defined in Sec. III, and $Q_{p r 5}$ described in the following paragraph. Configurations from the more successful of these are shown in Fig. 2, discussed in Sec. III.

A separate direction for improving the proxy has come from using GENE itself within the optimization loop. As noted in Ref. 4, doing nonlinear GENE runs to compute a FOM is currently much too computationally expensive. However, GENE can also be used in linear mode to compute the growth rates $\gamma_{\mathbf{k}}$, which is far faster, currently marginally fast enough to be practical. Thus, we have implemented a "GenE-in-Stellopt" (GiS) proxy $Q_{p r 5}$, of the same form as $Q_{p r 1 b}$, but where $\gamma_{\mathbf{k}}$ there is computed through linear GENE runs. This has the obvious advantage that the $\gamma_{\mathbf{k}}$ are computed without approximation, and generalization beyond the ITG-ae modes addressed by the present $Q_{p r 1}$ variants is limited only by the physics included in the GK code. In Sec. III we present early results from use of this proxy as well as the $Q_{p r 1}$ variants already introduced.

\section{EVOLVED CONFIGURATIONS}

Applying the proxies just discussed has resulted in a slowly growing set of configurations with reduced values of $Q_{p r}$, and often with substantially reduced $Q_{g k}$. The QA class has been the most studied. Shown in Fig. 2 are the poloidal cross-sections at the 2 symmetry planes $N \zeta=0, \pi$ of turbulence-reduced NCSX-derived configurations obtained using these proxies, compared with those of NCSX (in black). One notes that they fall into 3 subclasses, as judged by the plane at $N \zeta=\pi$, for which NCSX has a bullet-like form. For the 3 on the top row, this has evolved into a "breadslice" shape, including configurations QA_35q and QA_40n described in Refs. 4, 5, with $Q_{g k}$ down by factors of about 0.40 and 0.50 from that of NCSX, respectively, the latter also having slightly improved " $1 / \nu$ " NC transport $\left(\sim \epsilon_{e f}^{3 / 2} / \nu\right)$. On the second row, QA_111b, derived using proxy-1d, has a "bottle-like" cross-section, rather close to NCSX, with an $m=5$-like perturbation superposed on the bullet, and $Q_{g k}$ down by a factor of about 0.42 from NCSX, while maintaining excellent NC confinement. QA_98k is a second member of this bottle-like class, obtained using proxy-5. Its resemblance 
to QA_111b may be a result of the improved agreement between $Q_{g k}$ and $Q_{p r}$ for proxy-1d noted earlier. Finally, QA_107e2, obtained from proxy-1f, has an almost elliptical shape.

To find practical designs which one might want to build, one must impose additional criteria beyond just minimizing $Q_{p r}$ or $Q_{g k}$. The configuration must also not substantially degrade NC transport, have good stability properties, and have a shape which can be created with a feasible coil set. As a first step toward this end, the configurations shown in Fig. 2 have been "2nd-optimized", subjected to further optimization to meet these added criteria. The very pointed tips of the bean cross section $(N \zeta=0)$ of QA_107e2 probably make its implementation impractical. All 3 of the breadslice QAs are kink unstable, and slightly ballooning unstable, as is QA_98k. 2nd-optimizing, placing a larger weight on the kink- and ballooning contributions to $C^{2}$, we have obtained a breadslice configuration QA_121h which is satisfactory in all these respects, and with $Q_{g k}$ down from that for NCSX by a factor of about 0.65 (Fig. 3), with slightly worse but still excellent NC transport. Configuration QA_111b, the most promising QA found to date, is also kink and ballooning stable, with (as noted) $Q_{g k}$ down from NCSX by a factor of about 0.42 , and NC transport only $21 \%$ larger than for NCSX (which is far smaller than the turbulent transport). Moreover, its shape is not greatly different from that of NCSX, suggesting its coil set could resemble that of NCSX, and possibly a single device could be designed able to access both configurations. In Fig. 3 is shown the averaged $Q_{g k}$ versus time from nonlinear GenE runs, for several promising configurations, including these two 2nd-optimized ones.

As mentioned earlier, the QO/QI reference system W7X has proved the most resistant to having its $Q_{g k}$ reduced. As noted, it has long been recognized that STELLOPT's standard LM search algorithm, being local in character, makes the optimizer prone to stopping at suboptimal, local minima, and for this reason, STELlOPT was also equipped with a global, DE algorithm. [10] On the conjecture that the resistance of the W7X design to our turbulencereduction method was due to this weakness of the LM algorithm, an effort was made to apply the same method, but using the DE algorithm. The proxy used for this study was $Q_{p r 1 x}=$ $\left(g^{r r}\right)^{2} \kappa_{1} H\left(-\kappa_{1}\right)$, up to numerical factors close to the square of $Q_{p r 1 d} \sim g^{r r} \kappa_{1}^{1 / 2} H\left(-\kappa_{1}\right)$, hence with a similar topography in $\mathbf{Z}$-space. This has resulted in the discovery[11] of a new configuration, designated MPX, with $Q_{g k}$ about half that of W7X, and with $1 / \nu$ transport about a third of that in "high-mirror" W7X (W7X-hm). In Fig. 4 are shown, at left, a comparison of the $N \zeta=0, \pi$ cross-sections for W7X (black) and MPX (red), and at right, 
the profiles of field strength $B(\theta)$ along a field line for one poloidal transit. One notes for both the characteristic QO/QI signature in $B(\theta),[13]$ with the outboard ripple small compared with the inboard ripple.

As noted in Sec. I, the $Q_{g k}$ reduction for QA_35q and QA_40n was achieved by improving $\kappa_{1}$, especially near $\theta=0$, where the curvature drive is usually greatest. In contrast, $\kappa_{1}(\theta)$ for MPX is actually less favorable than that for W7X, but this is more than compensated by the $g^{r r}$ factor. A similar situation holds for the improvement of QA_111b over NCSX. Thus, the presence of the geometric factor $g^{r r}$ in $Q_{p r 1 d}$ and $Q_{p r 1 x}$ has given STELLOPT access to new families of configurations with reduced ITG turbulence not accessible via $Q_{p r 1}$ or $Q_{p r 1 b}$.

An earlier run on W7X with the LM algorithm using proxy-1d produced another configuration, QO_113f. This had substantially reduced $Q_{p r}$, but did not appreciably improve $Q_{g k}$. Nevertheless, to gain insight into why Stellopt with LM found QO_113f and not something closer to MPX, in Fig. 5 we use Stellopt in mapping mode, plotting the turbulent contribution $C_{t}^{2}=Q_{p r 1 d}^{2}$ to $C^{2}$ over the plane in shape-space $\mathbf{Z}$ defined by the $3 \mathrm{QO}$ reference configurations W7X, QO_113f (green) and MPX (blue). One sees that both $113 \mathrm{f}$ and MPX lie downhill in $Q_{p r}$ from W7X. Evident in the view shown is that MPX sits near the crest of a ridge, separating 2 basins in $Q_{p r}$, one in which $113 \mathrm{f}$ lies, and a second,still further away from W7X than MPX. While not apparent from the view taken for Fig. 5, the descent from W7X to $113 f$ lies in a slight gulley of $Q_{p r}$, probably explaining why STELLOPT with the LM followed that route.

In both basins, one notes that configurations are present with smaller $Q_{p r}$ than for either 113 or MPX. Indicated are configurations 130i and 130j, at the approximate $Q_{p r}$ minima for basins 1 and 2, respectively. Thus, the mapping capability can be used as an optimization tool in its own right, permitting one to find configurations with lower values of the cost function than the 2 configurations discovered with either the LM or the DE algorithms. These may then be used as starting points for further optimization via STELLOPT, which will in general search off the 2D subspace shown in Fig. 5. Study of this is currently underway. As shown in Fig. 6, GeNE runs corroborate the improvement from W7X for MPX, 130i, and $130 \mathrm{j}$ predicted by $Q_{p r 1 d}$, with $Q_{g k}$ down from that for W7X by a factor of about 0.89 for 130i, 0.48 for MPX, and 0.43 for 130j. The configurations over the plane in Fig. 5 have interpolated values of the volume-averaged plasma $\beta$ as well as of the boundary shape, with $\langle\beta\rangle=4.3 \%$ for W7X, $4.1 \%$ for 113f, and $0 \%$ for MPX, yielding $\langle\beta\rangle=1.1 \%$ for $130 \mathrm{i}$ and 
$1.7 \%$ for 130 j. For comparison, therefore, in Fig. 6 we also show the GENE results for the vacuum W7X, designated W7Xvac. One notes the comparison of the evolved configurations with this is still better than that for W7X at $\langle\beta\rangle=4.3 \%$.

Further progress has also been made for turbulence reduction for QHs, beyond the optimization using $Q_{p r 1}$ noted in Sec. I. This optimization is of special interest because HSX is the only $\mathrm{NC}$ transport optimized stellarator currently in operation, and therefore might provide early tests of these ideas. However, the configuration from that optimization, as well as a subsequent one done using $Q_{p r 1 d}$, would not fit in the HSX vacuum vessel. Moreover, heating in HSX is via ECRH, making electrons much hotter than ions, so that the dominant modes present are thought to be trapped electron modes (TEMs), rather than the ITG modes the current proxies address. On the former difficulty, it was observed that both evolved systems shared the qualitative feature that the axis excursion occurring each field period was reduced from that of the standard HSX "QHS" configuration. Accordingly, a configuration (QH_122a) was found by adjusting the currents in the existing HSX auxiliary coil set which both fit within the HSX vessel, and which possessed this reduced axis excursion. Nonlinear GENE runs on this found that QH_122a indeed showed a reduction from HSX in $Q_{g k}$ of about a factor of 2. However, the TEM versus ITG difficulty remains. A satisfactory comparison with experiment will accordingly need to await the development of proxies designed for optimizing for TEM rather than ITG turbulence.

\section{DISCUSSION}

In this work, we have described the progress made to date in finding turbulent-optimized toroidal configurations, using the STELlOPT optimizer and the GENE gyrokinetic code. We have successfully applied these tools to evolving toroidal designs to ones with appreciably reduced ITG turbulence, for each of the 4 classes of NC-optimized devices examined in our earlier GK survey.[12] The same method should also apply to stellarator/heliotron designs not optimized for NC transport, such as LHD (Large Helical Device)[14] in its standard configuration, or heliacs.

Improvement of the proxy function $Q_{p r}$ has been important in the progress made, with $Q_{p r 1 d}$ or its variant $Q_{p r 1 x}$ being the most effective analytic form found thus far. These have resulted in an enhanced set of geometric control knobs a designer has in reducing 
ITG turbulence, currently including the radial curvature $\kappa_{1}$, the local shear $s_{l}$ or perhaps instead the integrated shear $\Lambda=g^{r y} B_{a} / B \sim \int d z s_{l}$ and the metric coefficients $g^{r r}$ and $g^{y y}$. These have been identified through a combination of expectations from analytic theory and observation of numerical results. These are related, e.g., $g^{y y}=\left(1+\Lambda^{2}\right)\left(B / B_{a}\right)^{2} / g^{r r}$, and how many independent knobs there are, and what the correct dependence of $Q_{p r}$ on them is, is the subject of continuing study. Also requiring study is improved understanding of the shape deformations needed to produce a desirable distribution on a flux surface of these quantities, which STELlopt presently explores by brute force. Some insight into this has been achieved,[5] e.g., for how the breadslice class of QAs, or inboard-indented tokamaks boost average $\kappa_{1}$ on a surface. However, much remains to be explored.

The effectiveness of the method has been further enhanced, by both the added use of the global DE search algorithm, and also by using STELLOPT's mapping capability to better visualize the topography of the cost function and other key quantities over the search space.

All of the configurations described here, and all of the analytic proxies, have been directed toward reducing ITG turbulence. Applying the same general method to other important transport channels, e.g., TEM turbulence, will require either analytic proxies developed for that purpose, or an extended application of proxy-5, for which an improved, faster implementation will be needed, and should be feasible.

As discussed, we have also taken initial steps toward tuning the configurations found thus far to ones meeting the multiple constraints one needs for an experimental device. Experimental tests of these findings should be possible on various devices now in operation or soon to be.

\section{Acknowledgment}

This work supported by U.S.Department of Energy Contract No.DE-AC02-09CH11466.

[1] Reiman A, Fu G, Hirshman S, Ku L, Monticello D, Mynick H, Redi M, Spong D, Zarnstorff D, Blackwell B, Boozer A, Brooks A, Cooper W.A, Drevlak M, Goldston R, Harris J, Isaev M, Kessel C, Lin Z, Lyon J.F, Merkel P, Mikhailov M, Miner W, Nakajima N, Neilson G, 
Nührenberg C, Okamoto M, Pomphrey N, Reiersen W, Sanchez R, Schmidt J, Subbotin A, Valanju P, Watanabe K.Y, White R, 1999 Plasma Phys. Control. Fusion 41 B273.

[2] Jenko F, Dorland W, Kotschenreuther M, Rogers B.N, 2000 Phys. Plasmas 71904.

[3] Xanthopoulos P, Cooper W.A, Jenko F, Turkin Yu, Runov A, Geiger J, 2009 Phys. Plasmas 16082303.

[4] Mynick H, Pomphrey N, Xanthopoulos P, 2010 Phys. Rev. Letters 105095004.

[5] Mynick H, Pomphrey N, Xanthopoulos P, 2011 Phys. Plasmas 18056101.

[6] Neilson G.H, Zarnstorff M.C, Lyon J.F, the NCSX Team, 2002 Journal of Plasma and Fusion Research 78 214-219.

[7] Mynick H, Pomphrey N, Xanthopoulos P, Faber B, Lucia M, Rorvig M, Proceedings of the 24th IAEA Fusion Energy Conference (San Diego, CA, October, 2012), (International Atomic Energy Agency, Vienna, Austria), Paper TH/P2-04.

[8] Talmadge J.N, Sakaguchi V, Anderson F.S.B, Anderson D.T, Almagri A.F, 2001 Phys. Plasmas 85165 .

[9] Grieger G, Lotz W, Merkel P, et al., 1992 Phys. Fluids -B 42081.

[10] Mynick H, Pomphrey N, Ethier S, 2002 Phys. Plasmas 9869.

[11] Xanthopoulos P, Helander P,Mynick H, Turkin Yu, Jenko F, Görler T, Told D, Plunk G, Bird T, Proll, J, (submitted for publication, January, 2014).

[12] Mynick H.E, Xanthopoulos P, Boozer A.H, 2009 Phys.Plasmas 16110702.

[13] Mynick H.E, Chu T.K, Boozer A.H, 1982 Phys. Rev. Letters 48322.

[14] Fujiwara M, Takeiri Y, Shimozuma T, et al., 2000 Nucl. Fusion 40, 1157. 

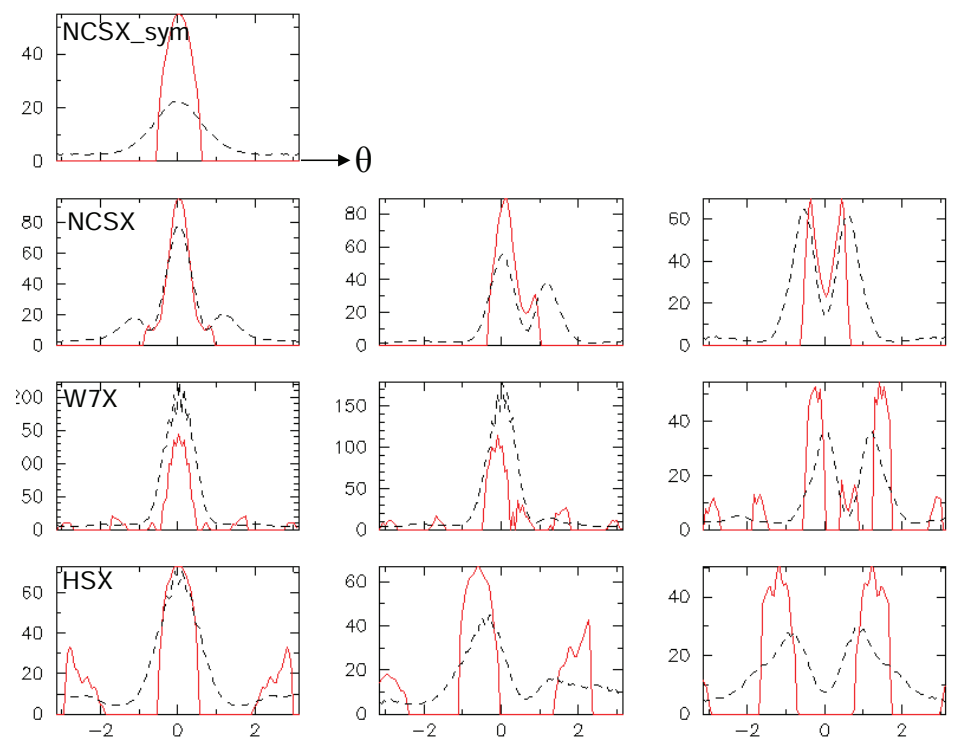

FIG. 1: (Color online) Comparison of $Q_{p r 1 d}$ (solid) and $Q_{g k}$ (dashed), along a field line for 1 poloidal transit, for each of the reference configurations. 1 flux tube is given for tokamak NCSX_sym, and 3 tubes for each of the 3 stellarators, along field lines with $N(\zeta-q \theta)=0, \pi / 2, \pi$. The $Q$ are given in standard GENE units of $\rho_{s}^{2} c_{s} p_{i} / a^{2}$.
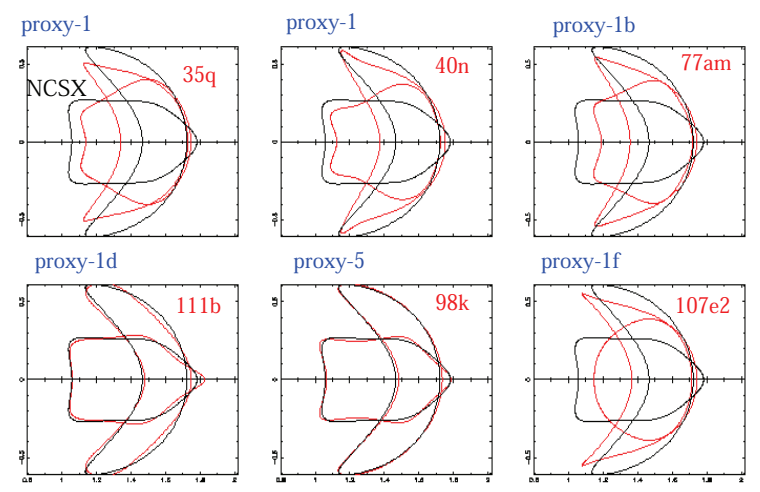

FIG. 2: (Color online) Poloidal cross sections of NCSX (black), and configurations evolved from it (red) having reduced turbulent transport. 


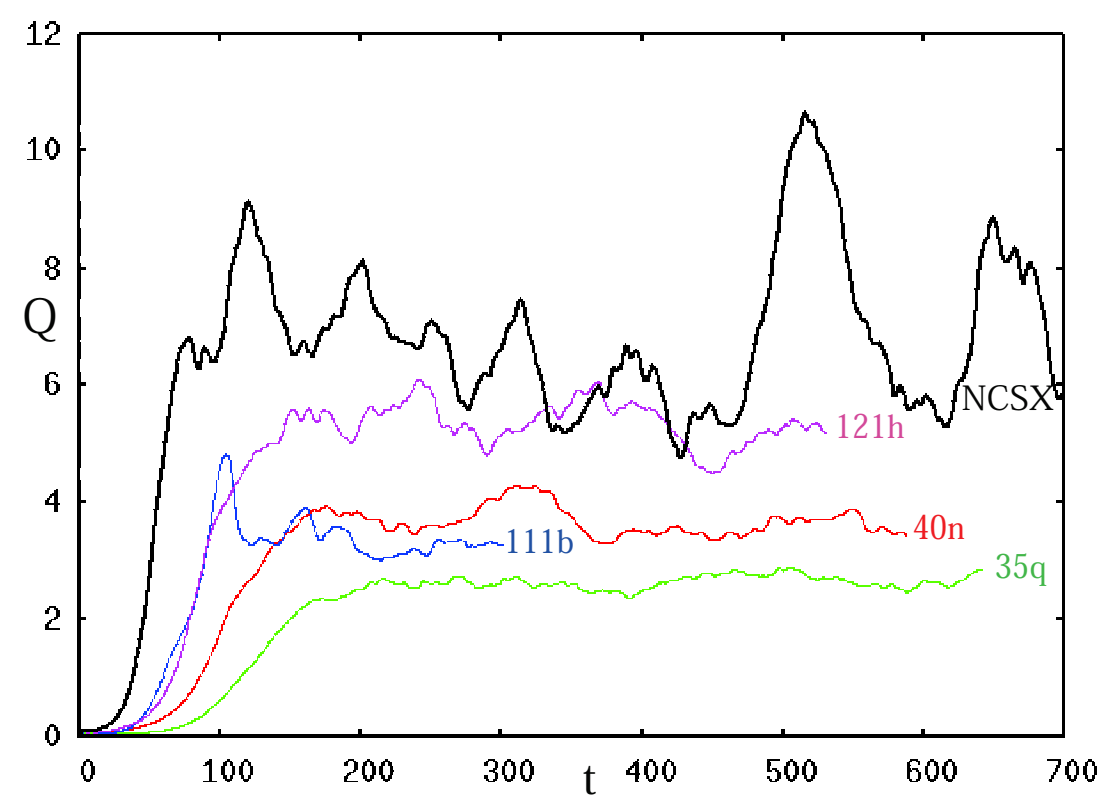

FIG. 3: (Color online) Averaged heat flux $Q_{g k}$ versus time from nonlinear GENE runs for NCSX (black) and several turbulence-reduced QA systems discussed in the text.
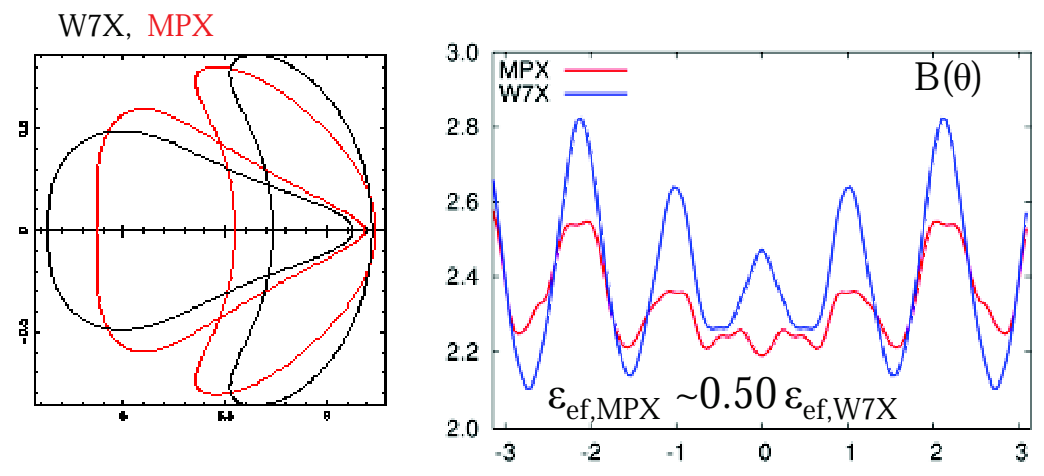

FIG. 4: (Color online) Left: Poloidal cross sections of W7X (black) and MPX (red). Right: Profiles of $B(\theta)$ along a field line for these 2 systems. 


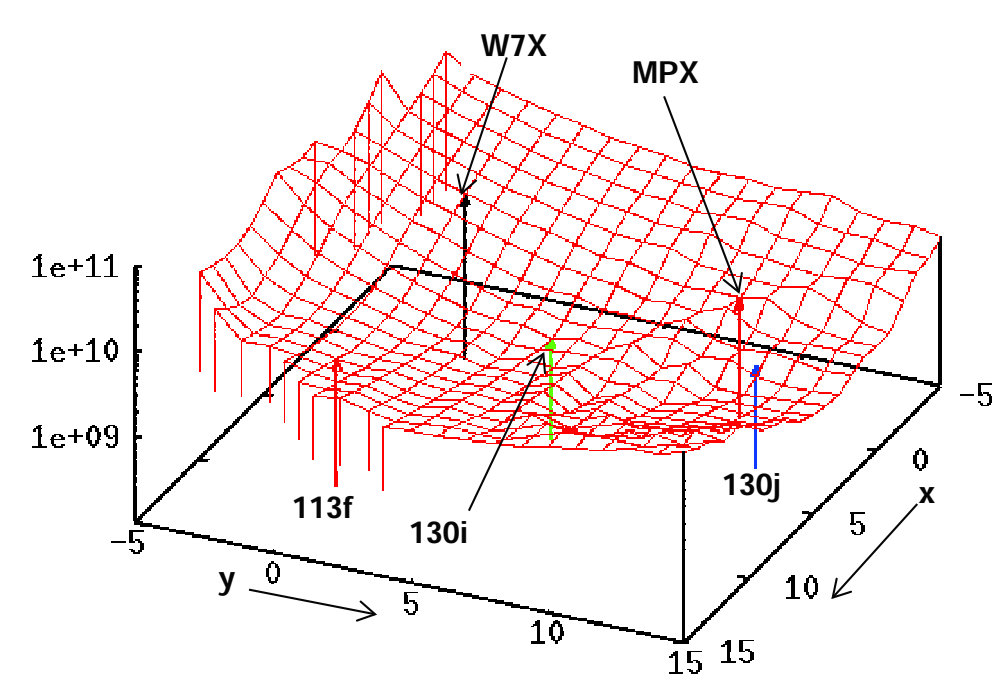

FIG. 5: (Color online) Plot of $C_{t}^{2}$ over the plane in shape-space defined by configurations W7X at $(x, y)=(0,0)$, QO_113f at $(10,0)$, and MPX at $(0,10)$. Also indicated are the positions of configurations $130 \mathrm{i}$ at $(4,5)$ and $130 \mathrm{j}$ at $(3,12)$, local minima in 2 adjoining basins.

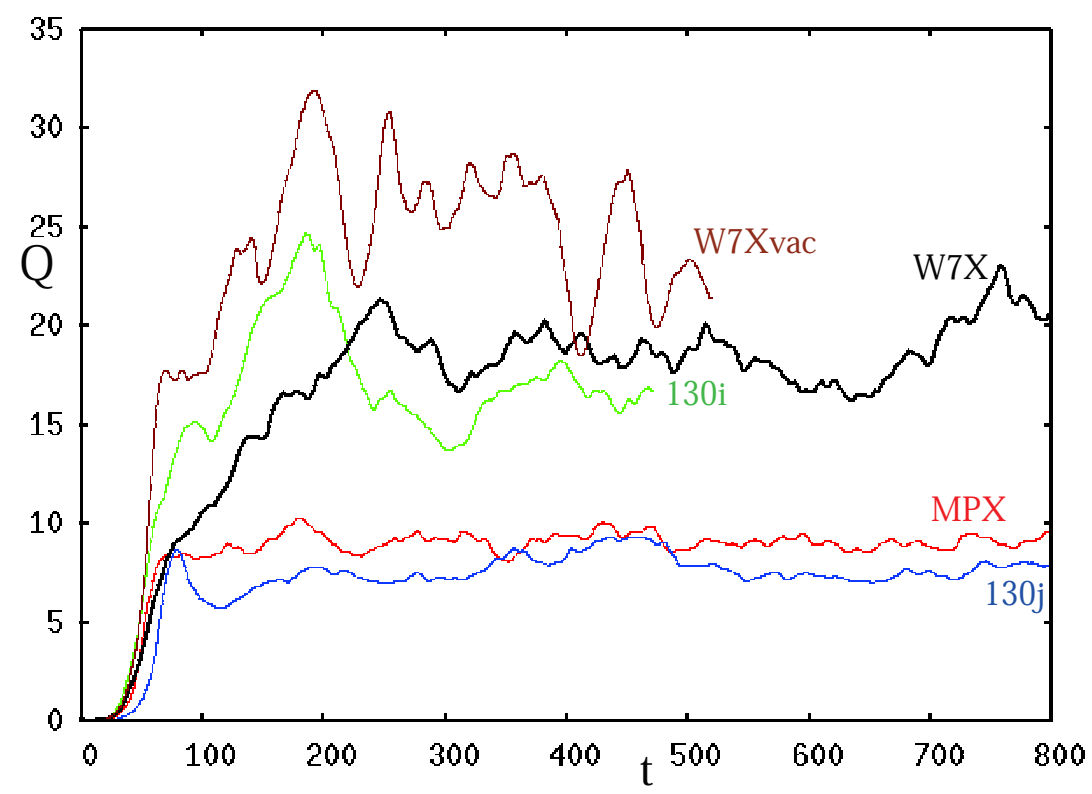

FIG. 6: (Color online) Averaged heat flux $Q_{g k}$ versus time from nonlinear GENE runs for W7X at volume-averaged $\langle\beta\rangle=0$ (labelled W7Xvac), at $\langle\beta\rangle=4.3 \%$, and QO configurations MPX, 130i and 130j evolved from W7X. 